УДК 616.891.7-053.81

Для цитирования: Петров А.А., Черняк Н.Б. Зависимость от компьютерных онлайн-игр как подтип интернет-аддикции (литературный обзор). Сибирский вестник психиатрии и наркологии. 2017; 4 (97): 82-88. https://doi.org/10.26617/18103111-2017-4(97)-82-88

\title{
Зависимость от компьютерных онлайн-игр как подтип интернет-аддикции (литературный обзор)
}

\author{
Петров А.А., Черняк Н.Б.
}

Иркутский государственный медииинский университет

Россия, 664003, Иркутск, ул. Красного Восстания, 1

\section{PEЗЮME}

Данный литературный обзор посвящен проблеме ведущего подтипа интернет-аддикции - зависимости от компьютерных онлайн-игр. Актуальность исследования обусловлена беспрецедентной клинической и социальной значимостью патологического использования сетевых компьютерных игр среди взрослых и подростков во всем мире. Приводятся положения о дискутабельности нозологической самостоятельности интернет-аддикции, описывается важность дифференциации различных подтипов интернет-зависимого поведения, подчеркивается весомость аддиктогенного потенциала компьютерных онлайн-игр. Последующее изложение касается эпидемиологии, критериев и инструментов диагностики, клиники, динамики, коморбидности и лечения интернет-аддикции и игровой компьютерной зависимости. В заключении подчеркивается важность включения зависимости от компьютерных онлайн-игр в раздел III DSM-V, так как последнее позволит прийти к консенсусу в плане верификации и терапии данного психопатологического феномена.

Ключевые слова: интернет-аддикция, зависимость от компьютерных игр, игровая компьютерная зависимость, интернет-игровое расстройство.

\section{ВВЕДЕНИЕ}

Интернет - это ресурс, доступный 24 часа в сутки для большинства населения земного шара [24]. Мировая сеть предоставляет ряд преимуществ при использовании её в коммерческих, социальных, научных, медицинских целях. Интернет-игры имеют позицию доминирующей формы развлечения для взрослых и подростков, поскольку цифровые технологии позволяют играть на самых разных устройствах [47]. Однако с быстрым развитием Интернета и его социальной интеграцией негативные последствия, связанные с глобальной сетью, приобретают крайнюю выраженность [51]. Некоторые сетевые пользователи становятся зависимыми от Интернета, что приводит к академической неуспеваемости, снижению производительности труда [19] и семейным конфликтам [41]. Несмотря на то что термин «интернет-зависимость» является спорным [43], это наиболее часто используемое понятие в международных изданиях. Вопрос о том, является ли Интернет самостоятельным объектом зависимости или средством облегчения аддиктивного поведения, всё ещё остается открытым [37]. Сохраняется актуальной дискуссия - является ли чрезмерное использование Интернета истинным психическим расстройством, обычным поведением или отражением построенной на средствах массовой информации моральной паники [25].

\section{ОБСУЖДЕНИЕ}

Следует отметить, что интернет-аддикты используют различные интернет-приложения или аддиктивные сайты. В подтверждении этому эмпирические данные свидетельствуют о дифференциации между генерализованной интернет-зависимостью и специфическими типами аддиктивного использо- вания Интернета [36]. M. Brand et al. (2014) предложен термин «расстройство специфического использования Интернета» (Specific Internet - Use Disorders»), который подразумевает наличие таких подтипов интернет-аддикции, как интернет-игровое расстройство (Internet Gaming Disorder), интернетгемблинг (internet gambling) и расстройство просмотра интернет-порнороликов (Internet Pornography Viewing Disorder) [17]. В свою очередь А.Ю. Егоров (2015) выделяет более расширенный ряд интернетаддиктов, а именно интернет-гемблеров, интернетгеймеров, интернет-трудоголиков, интернетсексоголиков, интернет-эротоголиков, интернетпокупателей и интернет-аддиктов отношений [3].

По сравнению с другими видами интернетзависимости пристрастие к компьютерным онлайниграм является наиболее значимым подтипом, проявляющим такую специфическую особенность, как игровое ролевое поведение в виртуальном мире [24]. В мае 2013 г. в связи с клинической и социальной значимостью зависимость от компьютерных онлайн-игр под названием «интернет-игрового расстройства» (Internet Gaming Disorder) была внесена в раздел III DSM-V как проблема, требующая дальнейших исследований [12]. Это стало первым случаем, когда зависимость от сетевых компьютерных игр была официально (хотя и условно) признана расстройством психического здоровья в психиатрической номенклатуре.

По данным эпидемиологических исследований зарубежных авторов, интернет-зависимостью страдает от 1 до 2\% населения земного шара $[10,14,39]$. При этом пристрастие к компьютерным онлайниграм является одним из наиболее популярных её видов [33]. 
Показатель распространенности игровой компьютерной зависимости в странах Западной Европы варьирует от 1,7 до $8,5 \%$ среди подростков $[32,38]$ и от 0,2 до $0,6 \%$ среди взрослых [26, 35]. В Китае зависимость от компьютерных игр определяется у 2,4\% молодого населения [34].

Точных данных о количестве интернет-аддиктов в России на сегодняшний день нет [3]. Отечественными исследователями выполнено ограниченное число работ, направленных на установление частоты интернет-аддикции в зависимости от компьютерных игр с использованием различных диагностических методик $[5,6,7]$. Анализ распространенности интернет-зависимого поведения среди московских подростков, проведенный В.Л. Малыгиным и др. [5], показал, что 4,3\% подростков имеют признаки сформированного интернет-зависимого поведения, а 29,3\% злоупотребляют интернет-ресурсами и относятся к группе риска в отношении проблемного использования Интернета.

Используя результаты анализа 3500 анкет-тестов K. Янг на интернет-зависимость, заполненных сетевыми пользователями, В.А. Лоскутова определила, что среди всех респондентов «здоровых» оказалось $74 \%$, «пограничных» - 24\%, «зависимых» - 2\% [6].

По данным исследования А.В. Урсу, А.В. Худякова (2009), проведенного среди учащихся 7-10-х классов общеобразовательных школ, студентов медицинской академии, энергоуниверситета и энергоколледжа г. Иванова, установлено, что признаки увлеченности компьютерными играми имеют почти половина школьников старших классов $(45,5 \%)$, треть учащихся колледжа $(29,3 \%)$ и каждый десятый студент вузов $(13,4 \%)$ [7].

Эмпирические исследования показывают, что симптомокомплекс интернет-аддикции соответствует критериям зависимости от психоактивных веществ [44]. В работах, посвященных интернет-аддикции (зависимости от компьютерных игр), применяются методы оценки, базирующиеся на диагностических критериях DSM-IV и DSM-V для наркомании и патологического гемблинга [22] или критериях МКБ-10 для зависимостей [45]. С момента возникновения понятий интернет-аддикции (зависимости от компьютерных игр) был разработан ряд психометрических шкал, насчитывающий в общей сложности 21 инструмент. Данные методики предназначены для идентификации интернет-аддикции (зависимости от компьютерных игр) в клинической и здоровой группах населения [29]. В качестве примера можно привести следующие опросники: Young's Internet Addiction Questionnaire, Ko's Internet Addiction Scale, Chen Internet Addiction Scale, Game Addiction Scale, Internet-Gaming Disorder 20 Test, Internet-Gaming Disorder Scale и ряд других. Значительная часть инструментов объемны и содержат более 15 вопросов. Наличие большого числа диагностических методик объясняется сохраняющимся дискуссионным статусом диагностических критериев зависимости от Интернета (компьютерных игр) [42].
Концептуализации их существенно различаются и в дополнение к этому границы разделения нормы от патологии варьируют, что препятствует научному и культуральному кросс-сравнению и ограничивает надежность исследований [30].

Диагностические критерии интернет-игрового расстройства по DSM-V наиболее близки по содержанию к диагностическим критериям патологического гемблинга и содержат 9 пунктов, которые должны быть реализованы клинически в течение последних 12 месяцев, а именно: (А) поглощенность интернет-играми, (В) симптомы отмены при отнятии Интернета, (C) толерантность, необходимость проводить всё большое количество времени за интернет-играми, (D) неудачные попытки контроля участия в интернет-играх, (Е) потеря интереса к хобби и развлечениям как результат, за исключением интернет-игр, (F) продолжение чрезмерного использования интернет-игр, несмотря на осознание психосоциальных проблем, (G) обман членов семьи, врачей или других относительно количества времени, затрачиваемого на интернет-игры, (Н) использование интернет-игр для избегания или облегчения негативного настроения, (I) потеря значимых отношений, работы, образовательных или карьерных возможностей из-за участия в интернет-играх [12].

На сегодняшний день в литературе представлены две отечественных клинико-динамических модели развития компьютерной зависимости (зависимости от компьютерных игр) [7, 9]. Так, Л.Н. Юрьевой, Т.Ю. Больбот (2006) определены и описаны три этапа развития компьютерной зависимости [9]. Первый этап - риск развития компьютерной зависимости; характеризуется повышением времени, проводимого за ПК, снижением продуктивности в работе; потерей ощущения времени, эмоциональным подъем при работе за компьютером, рост денежных расходов на его обслуживание, первые признаки социальной дезадаптации. Второй этап - сформированная компьютерная зависимость. Основными признаками, характерными для этого этапа, являются эмоционально-волевые нарушения и психическая зависимость. Третий этап - тотальная компьютерная зависимость (психическая и физическая зависимость, синдром актуализации компульсивного влечения). При этом наблюдаются признаки психической и физической зависимости, выражена социальная и семейная дезадаптация, безуспешны попытки контролировать работу за ПК. В структуре синдрома актуализации компульсивного влечения преобладают агрессивность, злобность, психомоторное возбуждение, депрессивные феномены, рассеянное внимание, непроизвольные «печатающие движения» пальцев рук. Возможно демонстративно-шантажное суицидальное поведение при попытке окружающих препятствовать компьютерной деятельности. Выражены физические симптомы: головная боль по типу мигрени, боль в позвоночнике, сухость в глазах, онемение и боль в пальцах кисти (синдром карпального канала). 
В свою очередь А.В. Урсу, А.В. Худяковым (2009) предложены следующие стадии компьютерной игровой зависимости: начальная (стадия напря- жения), средняя стадия (предельного напряжения), терминальная стадия (истощения) [7]. Их клиническое описание представлено в таблице.

\section{Стадии компьютерной игровой зависимости по А.В. Урсу, А.В. Худякову (2009)}

Т а б ли ц а

\begin{tabular}{|c|c|c|}
\hline \multicolumn{3}{|c|}{ Стадии компьютерной игровой зависимости по А.В. Урсу, А.В. Худякову (2009) } \\
\hline Начальная стадия (напряжения) & Средняя стадия (предельного напряжения) & Терминальная стадия (истощения) \\
\hline Стадия охранительного торможения & Стадия срыва охранительного торможения & Стадия истощения \\
\hline $\begin{array}{l}\text { Повышение толерантности (растет } \\
\text { среднее и максимальное время игры) }\end{array}$ & Максимальное нарастание толерантности & Снижение толерантности \\
\hline $\begin{array}{l}\text { В период игры эйфория периодически } \\
\text { сменяется выраженными отрицатель- } \\
\text { ными эмоциями, связанными с неуда- } \\
\text { чами в игре }\end{array}$ & $\begin{array}{l}\text { Скрытое (неосознаваемое) сокращение длитель- } \\
\text { ности эйфории вызывает стремление пройти за } \\
\text { раз «узловой момент» и побуждает к поиску } \\
\text { новых более мощных игр }\end{array}$ & $\begin{array}{l}\text { Явное уменьшение выраженности и продол- } \\
\text { жительности эйфории приводит к тому, что } \\
\text { игры становятся неинтересными, новые игры } \\
\text { быстро надоедают } \\
\end{array}$ \\
\hline $\begin{array}{l}\text { При воздержании наблюдается асте- } \\
\text { ническое состояние }\end{array}$ & $\begin{array}{l}\text { При воздержании проявляются признаки психи- } \\
\text { ческой зависимости: депрессия, дисфория, апа- } \\
\text { тия, раздражительность }\end{array}$ & $\begin{array}{l}\text { При воздержании возникает абстинентный } \\
\text { синдром, в структуру которого могут входить } \\
\text { вегетативные и соматические нарушения }\end{array}$ \\
\hline $\begin{array}{l}\text { Утрата контроля над временем в про- } \\
\text { цессе игры }\end{array}$ & Полная утрата контроля & $\begin{array}{l}\text { Псевдовосстановление контроля с рационали- } \\
\text { зацией }\end{array}$ \\
\hline $\begin{array}{l}\text { Постоянное навязчивое желание поиг- } \\
\text { рать }\end{array}$ & $\begin{array}{l}\text { Формируется интенсивное неодолимое влечение } \\
\text { к игре }\end{array}$ & $\begin{array}{l}\text { Сохраняется неодолимое влечение поиграть, } \\
\text { интенсивность снижается }\end{array}$ \\
\hline $\begin{array}{l}\text { На длительность игры оказывает вли- } \\
\text { яние внешняя ситуация, необходи- } \\
\text { мость выполнять свои обязанности }\end{array}$ & $\begin{array}{l}\text { Игровая деятельность приобретает постоянный } \\
\text { характер и прекращается не по воле играющего, } \\
\text { а в связи с конфликтами с близкими и принуди- } \\
\text { тельным отлучением от игры }\end{array}$ & $\begin{array}{l}\text { Игровая деятельность носит волнообразный } \\
\text { (запойный) характер и связана с появлением } \\
\text { новой игры или обновления. Прекращается } \\
\text { после прохождения игры или на фоне плохого } \\
\text { самочувствия }\end{array}$ \\
\hline Заострение черт личности & Изменение личности & $\begin{array}{l}\text { Снижение уровня личности, эмоционально- } \\
\text { волевое оскуднение }\end{array}$ \\
\hline $\begin{array}{l}\text { Временная заторможенность после } \\
\text { игры }\end{array}$ & Заторможенность и транзиторная дереализация & Заторможенность и дереализация \\
\hline Начальные явления астении & $\begin{array}{l}\text { Признаки субкомпенсации: выраженные астени- } \\
\text { ческие расстройства с явлениями вегетативной } \\
\text { дисфункции, тики, снохождения. Появляется } \\
\text { невротическая симптоматика }\end{array}$ & $\begin{array}{l}\text { Явления декомпенсации в виде обострения } \\
\text { хронических и провокации скрытых наслед- } \\
\text { ственно отягощенных заболеваний }\end{array}$ \\
\hline
\end{tabular}

Ведущими синдромами зависимости от компьютерных игр в рамках последней модели являются астенический, депрессивный, аддиктивный и синдром измененного состояния сознания [7].

Помимо клинико-динамических характеристик игровой компьютерной аддикции, внимание исследователей направлено и на сопутствующие психические расстройства. Так, по крайней мере расстройства Оси I DSM-IV имеют коморбидные отношения с патологическим использованием Интернета - синдромом дефицита внимания и гиперактивности [21], депрессией, тревожными расстройствами [11], злоупотреблением психоактивными веществами [28]. В исследовании Г.А. Джолдыгулова и др. (2005) определена возможность коморбидного сосуществования интернет-аддикции с шизофренией [2], в работе К. Wölfling et al. (2015) - с биполярным аффективным расстройством [46].

В исследованиях зарубежных авторов оценивается распространенность личностных расстройств среди пациентов с интернет-аддикцией (зависимостью от компьютерных игр) $[16,18,27,50]$. Представленность расстройств личности варьировала от 10 до 52\%. Среди интернет-аддиктов чаще наблюдались пограничное [1, 16, 20], нарциссическое [16], антисоциальное [16], избегающее [3, 20, 50] и зависимое [50] личностные расстройства. В исследовании S. Zadra et al. (2016) установлено, что пользователи с интернет-аддикцией достоверно чаще имеют личностное расстройство по сравнению с пользователями без интернет-аддикции [50].
Для оказания медицинской помощи пациентам с интернет-аддикцией (зависимостью от компьютерных игр) применяются терапевтические программы в виде трёх основных вариантов: психофармакотерапии, психотерапевтической коррекции, комбинации психотерапевтической методики и лекарственных препаратов.

В ряде исследований психофармакотерапия применялась изолированно $[4,13,15,22]$. Из препаратов использовались циталопрам 20-40 мг/сут в сочетании с кветиапином 50-200 мг/сут [13], эсциталопрам 10-20 мг/сут [22], флувоксамин 150-200 мг/сут, сертралин 150-200 мг/сут, флуоксетин 60 мг/сут, кломипрамин 200 мг/сут [15]. Во всех исследованиях наблюдалась редукция интернетаддиктивной симптоматики.

Многие работы посвящены описанию различных форм психологической терапии для лечения интернет-аддикции $[1,8,20,48,49]$. Когнитивноповеденческая терапия (КПТ) предложена в качестве ведущего метода коррекции, учитывая компульсивный характер расстройства. Принимая во внимание ежедневное и необходимое использование Интернета по сравнению с другими навязчивыми синдромами, доктором Кимберли Янг был разработан специализированный вид КПТ для лечения интернет-зависимости, получивший название «когнитивно-поведенческая психотерапия для интернетаддикции» (Cognitive Behavioral Therapy for Internet Addiction (CBT-IA) [49]. 
КПТ успешно сочетается с бупропионом замедленного высвобождения 150-300 мг/сут [31], клоназепамом 5 мг/сут и сертралином 50 мг/сут [40].

Из перечня отечественных психотерапевтических методик, разработанных для лечения интернетаддикции (зависимости от компьютерных игр) среди детей и подростков следует отметить психокоррекционную программу, разработанную Н.В. Вострокнутовым и др. (2009) на базе консультативно-диагностического отделения ГНЦ ССП им. В.П. Сербского и Центра профилактической медицины г. Москвы [1]. Данная программа включает три этапа: консультативно-диагностический, коррекционно-терапевтический и коррекционноподдерживающий. Ещё один отечественный метод коррекции компьютерной зависимости, осуществляемый в пределах проблемно-ориентированной психотерапии, предложенный А.Ф. Шайдулиной (2004), включает 4 этапа: диагностический, заключение психотерапевтического контракта, когнитивноповеденческий и социальной реабилитации [8].

\section{ЗАКЛЮЧЕНИЕ}

Проблема зависимости от компьютерных онлайн-игр и Интернета исключительно сложна, многоаспектна и недостаточно изучена. Несмотря на растущую значимость игровой компьютерной аддикции как глобальной угрозы здоровью подростков и молодежи, в настоящее время наблюдается отсутствие научно обоснованных мер по ведению пациентов с данным расстройством. Исследования по зависимости от компьютерных игр уже давно характеризуются непоследовательностью в терминологии, дефинициях и оценке. Хотя критерии интернетигрового расстройства не окончательно сформулированы, включение его в DSM-V приведет к более высокой степени стандартизации данного психопатологического феномена. Стандартизированный подход предоставит множество преимуществ, таких как улучшение в сопоставимости показателей распространенности и общих выводов во всех исследованиях. Возможный консенсус по определению интернет-игрового расстройства позволит производить более точную верификацию диагноза и повысит качество терапевтических программ в последующих клинических испытаниях.

\section{КОНФЛИКТ ИНТЕРЕСОВ}

Авторы заявляют об отсутствии конфликта интересов в связи с публикацией данной статьи.

\section{ИСТОЧНИК ФИНАНСИРОВАНИЯ}

Авторы заявляют об отсутствии финансирования при проведении исследования.

\section{ЛИТЕРАТУРА}

1. Вострокнутов Н.В., Пережогин Л.О. Зависимость от персонального компьютера, компьютерных игр и Интернета в детской психиатрической практике. Практическая медиична. 2009; 6 (38): 31-35.

2. Джолдыгулов Г.А., Гусманов Р.М., Шевченко Ю. К вопросу о механизмах формирования чрезмерной увлеченности компьютерными играми. Дискуссионные вопросы наркологии: профилактика, лечение и реабилитация: Материалы Российской научно-практической конференции. Иваново, 2005 $111-112$.
3. Егоров А.Ю. Современные представления об интернетаддикциях и подходах к их коррекции. Медицинская психология в России. 2015; 4 (33): 1-17.

4. Корнетов Н.А., Счастный Е.Д., Корнетов А.Н. Эффективность терапии депрессивных расстройств циталопрамом. Психиатрия и психофармакотерапия. 2001; 3 (4): 132-135.

5. Малыгин В.Л. и др. Интернет-зависимое поведение у подростков. Клиника, диагностика, профилактика. М.: Арсенал образования, 2010: 136

6. Лоскутова В.А. Интернет-зависимость как форма нехимических аддиктивных расстройств: диссертация. Новосибирск, 2004: 157.

7. Урсу А.В., Худяков А.В. Компьютерная игровая зависимость: клиника, динамика и эпидемиология. Психическое здоровье. 2009; 8 (39): 28-32.

8. Шайдулина А.Ф. Особенности клиники и лечения пациентов с патологической склонностью к азартным играм и компьютерной зависимостью: диссертация. СПб., 2004: 144.

9. Юрьева Л.Н., Больбот Т.Ю. Компьютерная зависимость: формирование, диагностика, коррекция и профилактика Днепропетровск: Пороги, 2006: 196.

10. Aboujaoude E. et al. Potential markers for problematic internet use: a telephone survey of 2,513 adults. CNS spectrums. 2006; 11 (10: 750-755.

11. Ahmadi J. et al. Prevalence of addiction to the internet, computer games, DVD, and video and its relationship to anxiety and depression in a sample of Iranian high school students. Iranian journal of psychiatry and behavioral sciences. 2014; 8 (2): 75.

12. American Psychiatric Association. Diagnostic and Statistical Manual of Mental Disorders (DSM-5). Arlington, VA: American Psychiatric Association, 2013.

13. Atmaca M. A case of problematic internet use successfully treated with an SSRI-antipsychotic combination. Progress in NeuroPsychopharmacology and Biological Psychiatry. 2007; 31 (4): 961-962.

14. Bakken I. J. et al. Internet addiction among Norwegian adults: a stratified probability sample study. Scandinavian journal of psychology. 2009; 50 (2): 121-127.

15. Bipeta R. et al. Diagnostic Stability of Internet Addiction in Obsessive-compulsive Disorder: Data from a Naturalistic One-year Treatment Study. Innovations in clinical neuroscience. 2015; 12.

16. Black D.W., Belsare G., Schlosser S. Clinical features, psychiatric comorbidity, and health-related quality of life in persons reporting compulsive computer use behavior. The Journal of clinical psychiatry. 1999; 60 (12): 839-844.

17. Brand M., Young K.S., Laier C. Prefrontal control and Internet addiction: a theoretical model and review of neuropsychological and neuroimaging findings. Frontiers in human neuroscience. 2014; 8: 375

18. Brand M., Young K.S., Laier C. Prefrontal control and Internet addiction: a theoretical model and review of neuropsychological and neuroimaging findings. Front. Hum. Neurosci. 8: 375. doi: 10.3389/fnhum.2014.00375.2014

19. Cheung L.M., Wong W.S. The effects of insomnia and internet addiction on depression in Hong Kong Chinese adolescents: an exploratory cross-sectional analysis. Journal of sleep research. 2011; 20 (2): 311-317.

20. Choo H., Gentile D.A., Sim T., Li D., Khoo A., Liau A.K. Pathological video gaming among Singapore an youth. Ann Acad Med Singapore. 2010; 39 (11): 822-829A.

21. Dalbudak E., Evren C. The relationship of Internet addiction severity with Attention Deficit Hyperactivity Disorder symptoms in Turkish University students; impact of personality traits, depression and anxiety. Comprehensive psychiatry. 2014; 55 (3): $497-$ 503.

22. Dell'Osso B. et al. Escitalopram in the treatment of impulsivecompulsive internet usage disorder: an open-label trial followed by a double-blind discontinuation phase. The Journal of clinical psychiatry. 2008; 69 (3): 452-456.

23. Demetrovics Z., Szeredi B., Rózsa S. The three-factor model of Internet addiction: The of the Problematic Internet Use Questionnaire. Behavior Research Methods. 2008; 40 (2): 563-574.

24. Dong G., Huang J., Du X. Alterations in regional homogeneity of resting-state brain activity in internet gaming addicts. Behavioral and Brain Functions. 2012; 8 (1): 41. 
25. Ferguson C.J., Coulson M., Barnett J. A meta-analysis of pathological gaming prevalence and comorbidity with mental health, academic and social problems. Journal of psychiatric research. 2011; 45 (12): 1573-1578.

26. Festl R., Scharkow M., Quandt T. Problematic computer game use among adolescents, younger and older adults. Addiction. 2013; 108 (3): 592-599.

27. Floros G. et al. Comorbidity of psychiatric disorders with Internet addiction in a clinical sample: the effect of personality, defense style and psychopathology. Addictive behaviors. 2014; 39 (12): 1839-1845.

28. Heo J. et al. Addictive internet use among Korean adolescents: a national survey. PLoS One. 2014; 9 (2): e87819.

29. Kuss D.J. et al. Internet addiction: a systematic review of epidemiological research for the last decade. Current pharmaceutical design. 2014; 20 (25): 4026-4052.

30. Kuss D.J., van Rooij A., Shorter G.W., Griffiths M.D., van de Mheen D. Internet addiction in adolescents: Prevalence and risk factors. Computers in Human Behavior. 2013.29 (5): 1987-1996.

31. Kim S.M. et al. Combined cognitive behavioral therapy and bupropion for the treatment of problematic on-line game play in adolescents with major depressive disorder. Computers in human behavior. 2012; 28 (5): 1954-1959.

32. King D.L. et al. Clinical features and axis I comorbidity of Australian adolescent pathological Internet and video game users. Australian and New Zealand Journal of Psychiatry. 2013; 47 (110: 1058-1067.

33. Kishi T. et al. Serotonin $1 \mathrm{~A}$ receptor gene and major depressive disorder: an association study and meta-analysis. Journal of human genetics. 2009; 54 (11): 629-633.

34. Lam L.T. et al. The association between internet addiction and self-injurious behaviour among adolescents. Injury prevention. 2009; 15 (6): 403-408.

35. Mentzoni R.A. et al. Problematic video game use: estimated prevalence and associations with mental and physical health. Cyberpsychology, behavior, and social networking. 2011; 14 (10): 591-596.

36. Montag C. et al. Is it meaningful to distinguish between generalized and specific Internet addiction? Evidence from a crosscultural study from Germany, Sweden, Taiwan and China. Asia Pacific Psychiatry. 2015; 7 (1): 20-26.

37. Petry N.M., O'Brien C.P. Internet gaming disorder and the DSM-5. Addiction. 2013; 108 (7): 1186-1187.

38. Rehbein F. et al. Prevalence and risk factors of video game dependency in adolescence): results of a German nationwide survey. Cyberpsychology, Behavior, and Social Networking. 2010; 13 (3): 269-277.
39. Rumpf H.J. et al. Occurence of internet addiction in a general population sample): a latent class analysis. European addiction research. 2013; 20 (4): 159-166.

40. Santos V., EgidioNardi A., Lucia Spear King A. Treatment of internet addiction in patient with panic disorder and obsessive compulsive disorder): a case report. CNS \& Neurological Disorders-Drug Targets (Formerly Current Drug Targets-CNS \& Neurological Disorders). 2015; 14 (3): 341-344.

41. Şenormancı Ö. et al. Attachment and family functioning in patients with internet addiction. General hospital psychiatry. 2014; 36 (2): 203-207.

42. Shapira N.A. et al. Psychiatric features of individuals with problematic internet use. Journal of affective disorders. 2000; 57 (1): 267-272.

43. Starcevic V. Is Internet addiction a useful concept? Australian and New Zealand Journal of Psychiatry. 2013; 47 (1): 16-19.

44. Thalemann R., Wölfling K., Grüsser S.M. Specific cue reactivity on computer game-related cues in excessive gamers. Behavioral neuroscience. 2007; 121 (3): 614.

45. Walther B., Morgenstern M., Hanewinkel R. Co-occurrence of addictive behaviours): personality factors related to substance use, gambling and computer gaming. European addiction research. 2012; 18 (4): 167-174.

46. Wölfling K. et al. Bipolar spectrum disorders in a clinical sample of patients with Internet addiction): Hidden comorbidity or differential diagnosis? Journal of behavioral addictions. 2015; 4 (2): 101-105.

47. Xu C.S., Chen J.S., Adelman R.A. Focus: Addiction: Video Game Use in the Treatment of Amblyopia: Weighing the Risks of Addiction. The Yale journal of biology and medicine. 2015; 88 (3): 309.

48. Young K.S. Cognitive behavior therapy with Internet addicts): treatment outcomes and implications. Cyber Psychology \& Behavior. 2007; 10 (5): 671-679.

49. Young K.S. Treatment outcomes using CBT-IA with Internetaddicted patients. Journal of behavioral addictions. 2013; 2 (4): 209-215.

50. Zadra S. et al. The association between Internet addiction and personality disorders in a general population-based sample. Journal of Behavioral Addictions. 2016; 5 (4): 691-699.

51. Zhou Z., Yuan G., Yao J. Cognitive biases toward Internet gamerelated pictures and executive deficits in individuals with an Internet game addiction. PloS One. 2012; 7 (11): e48961.

Поступила в редакцию 28.08.2017 Утверждена к печати 30.10.2017

Петров Александр Александрович, аспирант кафедры психиатрии и медицинской психологии.

Черняк Наталья Борисовна, к.м.н., ассистент кафедры психиатрии и медицинской психологии.

Черняк Наталья Борисовна, cherniakn@yandex.ru

УДК 616.891.7-053.81

For citation: Petrov A.A., Chernyak N.B. Dependence on computer online games as a subtype of internet addiction (literature review). Siberian Herald of Psychiatry and Addiction Psychiatry. 2017; 4 (97): 82-88. https://doi.org/10.26617/1810-3111-20174(97)-82-88

\section{Dependence on computer online games as a subtype of internet addiction (literature review)}

\section{Petrov A.A., Chernyak N.B.}

Irkutsk State Medical University

Krasnogo Vosstaniya Street 1, 664003, Irkutsk, Russian Federation

\section{ABSTRACT}

This literature review is devoted to the problem of the leading subtype of the Internet addiction - dependence on computer online games. The relevance of the study is caused by the unprecedented clinical and social significance of the pathological use of networked computer games among adults and adolescents worldwide. Provisions are made about the discreteness of the nosological independence of the Internet addiction, the importance of differentiating various subtypes of Internet-dependent behavior is de- 
scribed, severity of the addictogenic potential of computer online games is emphasized. The following presentation concerns epidemiology, criteria and diagnostic tools, clinics, dynamics, comorbidity and treatment of Internet addiction / game computer dependency. In conclusion, the importance of including dependence on computer online games in the third section of the DSM-V is emphasized, since the latter will lead to a consensus in terms of verification and therapy of this psychopathological phenomenon.

Keywords: Internet addiction, dependence on computer games, computer game dependency, internet gaming disorder.

\section{REFERENCES}

1. Vostroknutov N.V., Perezhogin L.O. Zavisimost ot personalnogo kompyutera, kompyuternyih igr i Interneta $\mathrm{v}$ detskoy psihiatricheskoy praktike [Dependence on the personal computer, computer games and Internet in children's psychiatric practice]. Praktich eskaya meditsina - Practical Medicine. 2009; 6 (38): 31-35 (in Russian).

2. Dzholdyigulov G.A., Gusmanov R.M., Shevchenko Yu. K voprosu o mehanizmah formirovaniya chrezmernoy uvlechennosti kom-pyuternyimi igrami [To the question of the mechanisms of formation of excessive enthusiasm for computer games]. Diskussionnyie voprosyi narkologii: profilaktika, lechenie i reabilitatsiya: Materialyi Rossiyskoy nauchno-prakticheskoy konferentsii. Ivanovo, 2005: 111-112 (in Russian).

3. Egorov A.Yu. Sovremennyie predstavleniya ob internetaddiktsiyah i podhodah $\mathrm{k}$ ih korrektsii [Modern ideas about Internet addictions and approaches to their Correction]. Meditsinskaya psihologiya $v$ Rossii-Medical Psychology in Russia. 2015; 4 (33) 1-17 (in Russian).

4. Kornetov N.A., Schastnyiy E.D., Kornetov A.N. Effektivnost terapii depressivnyih rasstroystv tsitalopramom [Efficiency of therapy of depressive frustration citalopram]. Psihiatriya $i$ psihofarmakoterapiya - Psychiatry and Psychopharmacotherapy. 2001; 3 (4): 132-135.

5. Malyigin V.L. i dr. Internet-zavisimoe povedenie u podrostkov. Klinika, diagnostika, profilaktika [Internet-addiction behavior in adolescents. Clinic, diagnosis, prevention]. M.: Arsenal obrazovaniya, 2010: 136 (in Russian).

6. Loskutova V.A. Internet-zavisimost kak forma nehimicheskih addiktivnyih rasstroystv: dissertatsiya [Internet addiction as a form of non-chemical addictive]. Novosibirsk, 2004): 157 (in Russian)

7. Ursu A.V., Hudyakov A.V. Kompyuternaya igrovaya zavisimost): klinika, dinamika i epidemiologiya [Computer game addiction: clinic, dynamics and epidemiology]. Psihicheskoe zdoroveMental Health. 2009; 8 (39): 28-32 (in Russian).

8. Shaydulina A.F. Osobennosti kliniki i lecheniya patsientov s patologicheskoy sklonnostyu k azartnyim igram i kompyuternoy zavisimostyu): dissertatsiya [Features of the clinic and treatment of patients with pathological predisposition to gambling and computer addiction]. Sankt-Peterburg, 2004: 144 (in Russian).

9. Yureva L.N., Bolbot T.Yu. Kompyuternaya zavisimost: formirovanie, diagnostika, korrektsiya i profilaktika [Computer addiction: formation, diagnosis, correction and prevention]. Dnepropetrovsk: Porogi, 2006: 196 (in Russian).

10. Aboujaoude E. et al. Potential markers for problematic interne use: a telephone survey of 2,513 adults. CNS spectrums. 2006; 11 (10: 750-755.

11. Ahmadi J. et al. Prevalence of addiction to the internet, computer games, DVD, and video and its relationship to anxiety and depression in a sample of Iranian high school students. Iranian journal of psychiatry and behavioral sciences. 2014; 8 (2): 75.

12. American Psychiatric Association. Diagnostic and Statistical Manual of Mental Disorders (DSM-5). Arlington, VA: American Psychiatric Association, 2013.

13. Atmaca M. A case of problematic internet use successfully treated with an SSRI-antipsychotic combination. Progress in NeuroPsychopharmacology and Biological Psychiatry. 2007; 31 (4): 961-962.

14. Bakken I. J. et al. Internet addiction among Norwegian adults: a stratified probability sample study. Scandinavian journal of psychology. 2009; 50 (2): 121-127.

15. Bipeta R. et al. Diagnostic Stability of Internet Addiction in Obsessive-compulsive Disorder: Data from a Naturalistic One-year Treatment Study. Innovations in clinical neuroscience. 2015; 12.

16. Black D.W., Belsare G., Schlosser S. Clinical features, psychiatric comorbidity, and health-related quality of life in persons reporting compulsive computer use behavior. The Journal of clinical psychiatry. 1999; 60 (12): 839-844.

17. Brand M., Young K.S., Laier C. Prefrontal control and Internet addiction: a theoretical model and review of neuropsychological and neuroimaging findings. Frontiers in human neuroscience. 2014; 8: 375 .

18. Brand M., Young K.S., Laier C. Prefrontal control and Internet addiction: a theoretical model and review of neuropsychological and neuroimaging findings. Front. Hum. Neurosci. 8: 375. doi: 10.3389/fnhum.2014.00375.2014

19. Cheung L.M., Wong W.S. The effects of insomnia and internet addiction on depression in Hong Kong Chinese adolescents: an exploratory cross-sectional analysis. Journal of sleep research. 2011; 20 (2): 311-317.

20. Choo H., Gentile D.A., Sim T., Li D., Khoo A., Liau A.K. Pathological video gaming among Singapore an youth. Ann Acad Med Singapore. 2010; 39 (11): 822-829A.

21. Dalbudak E., Evren C. The relationship of Internet addiction severity with Attention Deficit Hyperactivity Disorder symptoms in Turkish University students; impact of personality traits, depression and anxiety. Comprehensive psychiatry. 2014; 55 (3): 497503.

22. Dell'Osso B. et al. Escitalopram in the treatment of impulsivecompulsive internet usage disorder: an open-label trial followed by a double-blind discontinuation phase. The Journal of clinical psychiatry. 2008; 69 (3): 452-456.

23. Demetrovics Z., Szeredi B., Rózsa S. The three-factor model of Internet addiction: The of the Problematic Internet Use Questionnaire. Behavior Research Methods. 2008; 40 (2): 563-574.

24. Dong G., Huang J., Du X. Alterations in regional homogeneity of resting-state brain activity in internet gaming addicts. Behavioral and Brain Functions. 2012; 8 (1): 41.

25. Ferguson C.J., Coulson M., Barnett J. A meta-analysis of pathological gaming prevalence and comorbidity with mental health, academic and social problems. Journal of psychiatric research. 2011; 45 (12): 1573-1578.

26. Festl R., Scharkow M., Quandt T. Problematic computer game use among adolescents, younger and older adults. Addiction. 2013; 108 (3): 592-599.

27. Floros G. et al. Comorbidity of psychiatric disorders with Internet addiction in a clinical sample: the effect of personality, defense style and psychopathology. Addictive behaviors. 2014; 39 (12): 1839-1845.

28. Heo J. et al. Addictive internet use among Korean adolescents: a national survey. PLoS One. 2014; 9 (2): e87819.

29. Kuss D.J. et al. Internet addiction: a systematic review of epidemiological research for the last decade. Current pharmaceutical design. 2014; 20 (25): 4026-4052.

30. Kuss D.J., van Rooij A., Shorter G.W., Griffiths M.D., van de Mheen D. Internet addiction in adolescents: Prevalence and risk factors. Computers in Human Behavior. 2013. 29 (5): 1987-1996.

31. Kim S.M. et al. Combined cognitive behavioral therapy and bupropion for the treatment of problematic on-line game play in adolescents with major depressive disorder. Computers in human behavior. 2012; 28 (5): 1954-1959.

32. King D.L. et al. Clinical features and axis I comorbidity of Australian adolescent pathological Internet and video game users. Australian and New Zealand Journal of Psychiatry. 2013; 47 (110: 1058-1067.

33. Kishi T. et al. Serotonin $1 \mathrm{~A}$ receptor gene and major depressive disorder: an association study and meta-analysis. Journal of human genetics. 2009; 54 (11): 629-633.

34. Lam L.T. et al. The association between internet addiction and self-injurious behaviour among adolescents. Injury prevention. 2009; 15 (6): 403-408.

35. Mentzoni R.A. et al. Problematic video game use: estimated prevalence and associations with mental and physical health. Cyberpsychology, behavior, and social networking. 2011; 14 (10): 591-596. 
36. Montag C. et al. Is it meaningful to distinguish between generalized and specific Internet addiction? Evidence from a crosscultural study from Germany, Sweden, Taiwan and China. Asia Pacific Psychiatry. 2015; 7 (1): 20-26.

37. Petry N.M., O'Brien C.P. Internet gaming disorder and the DSM-5. Addiction. 2013; 108 (7): 1186-1187.

38. Rehbein F. et al. Prevalence and risk factors of video game dependency in adolescence): results of a German nationwide survey. Cyberpsychology, Behavior, and Social Networking. 2010; 13 (3): 269-277.

39. Rumpf H.J. et al. Occurence of internet addiction in a general population sample): a latent class analysis. European addiction research. 2013; 20 (4): 159-166.

40. Santos V., EgidioNardi A., Lucia Spear King A. Treatment of internet addiction in patient with panic disorder and obsessive compulsive disorder): a case report. CNS \& Neurological Disor ders-Drug Targets (Formerly Current Drug Targets-CNS \& Neurological Disorders). 2015; 14 (3): 341-344.

41. Şenormancı Ö. et al. Attachment and family functioning in patients with internet addiction. General hospital psychiatry. 2014; 36 (2) 203-207.

42. Shapira N.A. et al. Psychiatric features of individuals with problematic internet use. Journal of affective disorders. 2000; 57 (1): 267-272.

43. Starcevic V. Is Internet addiction a useful concept? Australian and New Zealand Journal of Psychiatry. 2013; 47 (1): 16-19.

44. Thalemann R., Wölfling K., Grüsser S.M. Specific cue reactivity on computer game-related cues in excessive gamers. Behavioral neuroscience. 2007; 121 (3): 614
45. Walther B., Morgenstern M., Hanewinkel R. Co-occurrence of addictive behaviours): personality factors related to substance use, gambling and computer gaming. European addiction research. 2012; 18 (4): 167-174.

46. Wölfling $\mathrm{K}$. et al. Bipolar spectrum disorders in a clinical sample of patients with Internet addiction): Hidden comorbidity or differential diagnosis? Journal of behavioral addictions. 2015; 4 (2): 101-105.

47. Xu C.S., Chen J.S., Adelman R.A. Focus: Addiction: Video Game Use in the Treatment of Amblyopia: Weighing the Risks of Addiction. The Yale journal of biology and medicine. 2015; 88 (3): 309.

48. Young K.S. Cognitive behavior therapy with Internet addicts: treatment outcomes and implications. Cyber Psychology \& Behavior. 2007; 10 (5): 671-679.

49. Young K.S. Treatment outcomes using CBT-IA with Internetaddicted patients. Journal of behavioral addictions. 2013; 2 (4): 209-215.

50. Zadra S. et al. The association between Internet addiction and personality disorders in a general population-based sample. Journal of Behavioral Addictions. 2016; 5 (4): 691-699.

51. Zhou Z., Yuan G., Yao J. Cognitive biases toward Internet gamerelated pictures and executive deficits in individuals with an Internet game addiction. PloS One. 2012; 7 (11): e48961.

Received August 28.2017

Accepted October 30.2017

Petrov Alexander A., post-graduate student of Psychiatry and Medical Psychology Department, Irkutsk State Medical University, Irkutsk, Russian Federation.

Chernyak Natalia B., PhD, assistant of Psychiatry and Medical Psychology Department, Irkutsk State Medical University, Irkutsk, Russian Federation.

Chernyak Natalia B., cherniakn@yandex.ru 